(C) The Authors 2016. This is an Open Access article, distributed under the terms of the Creative

Commons Attribution licence (http://creativecommons.org/licenses/by/4.0/), which permits

unrestricted re-use, distribution, and reproduction in any medium, provided the original work is properly cited.

\title{
The progression of coeliac disease: its neurological and psychiatric implications
}

\author{
Giovanna Campagna, Mirko Pesce*, Raffaella Tatangelo, Alessia Rizzuto, Irene La Fratta and Alfredo Grilli \\ Medicine and Health Science School, Università "G. d'Annunzio", Via dei Vestini, 31, 66100 Chieti CH, Italy
}

\section{Abstract}

The aim of the paper is to show the various neurological and psychiatric symptoms in coeliac disease (CD). CD is a T cell-mediated, tissuespecific autoimmune disease which affects genetically susceptible individuals after dietary exposure to proline- and glutamine-rich proteins contained in certain cereal grains. Genetics, environmental factors and different immune systems, together with the presence of auto-antigens, are taken into account when identifying the pathogenesis of CD. CD pathogenesis is related to immune dysregulation, which involves the gastrointestinal system, and the extra-intestinal systems such as the nervous system, whose neurological symptoms are evidenced in CD patients. A gluten-free diet (GFD) could avoid cerebellar ataxia, epilepsy, neuropathies, migraine and mild cognitive impairment. Furthermore, untreated CD patients have more symptoms and psychiatric co-morbidities than those treated with a GFD. Common psychiatric symptoms in untreated CD adult patients include depression, apathy, anxiety, and irritability and schizophrenia is also common in untreated CD. Several studies show improvement in psychiatric symptoms after the start of a GFD. The present review discusses the state of the art regarding neurological and psychiatric complications in $\mathrm{CD}$ and highlights the evidence supporting a role for GFD in reducing neurological and psychiatric complications.

Key words: Coeliac disease: Gluten-free diet: Neurological complications: Cognitive impairment: Psychiatric disorders

\section{Introduction}

A new definition of coeliac disease $(\mathrm{CD})$ has recently been presented by The European Society for Pediatric Gastroenterology, Hepatology and Nutrition, describing it as ' $\ldots$ an immune-mediated systemic disorder elicited by gluten and related prolamines in genetically susceptible individuals and characterized by a variable combination of gluten-dependent manifestations, CD-specific antibodies, HLA-DQ2 or HLA-DQ8 haplotypes, and enteropathy ${ }^{\text {(1) }}$. Transglutaminase (tTG), a multifunctional enzyme tissue, has been widely reported to be a major auto-antigen in CD. The identification of IgA autoantibodies directed towards this self-protein is crucial in the diagnosis of CD. The CD pathogenesis has also been reported to strongly involve tTG through the deamidation of gluten peptides that, once modified, are more easily presented to the immune system via human leucocyte antigen (HLA)-DQ2 or via DQ8 molecules. Gluten peptides reach the lamina propria and are presented to the T cells. Subsequently, several biological processes are triggered, leading to an increased $\mathrm{T}$ cell influx, crypt hyperplasia, and villous shortening in the proximal small intestine. As far as the prevalence of $\mathrm{CD}$ is concerned, many investigations are in accordance in evidencing that this disease is becoming more frequent in several geographical areas.
Nowadays, CD epidemiology is characterised not only by an increase in the cases reported in Northern Europe and the USA (historical areas of the spreading of CD), but also by its manifestation in different and new regions, such as Asian countries. This could be explained by the significant changes in the eating habits of these populations, who have increased the use of gluten especially during their childhood ${ }^{(2)}$. The way in which the disease changes and its clinical manifestations have been reported over time. Diarrhoea and malabsorption are not as frequent as they used to be in the initial stage of $\mathrm{CD}$, among both adults and children. On the other hand, there has been an increase in non-specific signs and atypical manifestations ${ }^{(3)}$. Furthermore, CD signs and symptoms are no longer limited only to the gastrointestinal tract, as reported in the past, but more than half of adult patients show extra-intestinal manifestations that are also expected to be improved by a gluten-free diet (GFD) ${ }^{(4)}$. To date, the only treatment for $\mathrm{CD}$ with complete remission of symptoms is a lifetime diet with the total elimination of gluten. Even the ingestion of small amounts of gluten can cause major disruptions; therefore following a gluten-free regimen can lead to the relief of the symptoms, the normalisation of histological and laboratory findings and a decrease in the risk of CD's associative complications. For this reason, $\mathrm{CD}$ should be considered as a complex disease as well as a multifactorial pathogenesis to be

Abbreviations: CD, coeliac disease; CNS, central nervous system; EEG, electroencephalogram; GFD, gluten-free diet; HLA, human leucocyte antigen; IEC, intestinal epithelial cell; IEL, intra-epithelial lymphocyte; IFN, interferon; NK, natural killer; QOL, quality of life; SBD, sleep breathing disorders; SCZ, schizophrenia; tTG, transglutaminase.

* Corresponding author: Mirko Pesce, email mirkopesce@unich.it 
investigated from a genetic, biological and environmental point of view also considering the nervous system and its implications. This review aims to describe the detailed research on CD, highlighting its findings in different domains (genetics, environmental triggers and immune pathogenesis), focusing on more recent areas of investigation connected with neurological complications, cognitive impairment, psychiatric disorders and the impact of quality of life (QOL) in coeliac patients. Research undertaken demonstrates that good adherence to a GFD may also be extremely beneficial to both neurological and psychiatric symptoms.

Notably, the role of the GFD in subclinical neurological abnormalities has not been assessed and the impact on histological course needs further investigation. It is well established that the GFD represents the most important aspect of the management of $\mathrm{CD}$ patients and it is the only treatment that allows the prevention of several associated malignant and nonmalignant complications, including neurological and psychiatric diseases.

\section{Pathogenesis}

\section{Genetics}

Recent studies in CD show that the disease is the interaction between genetic, environmental and immunological factors. The strongest and best-characterised genetic susceptibility factors in CD are HLA, especially the so-called DQ genes. The evidence of a genetic component in $\mathrm{CD}$ is best illustrated by the strong dependence on the presence of the HLA-DQ2 and HLADQ8 haplotypes ${ }^{(5-7)}$, most probably due to their physiochemical properties ${ }^{(8,9)}$ and binding of specific peptides dominated by tissue tTG2. A Europe-wide collaborative study reported that only $0.4 \%$ of $\mathrm{CD}$ patients were neither DQ2 (including the half heterodimer) nor DQ8 carriers ${ }^{(10)}$. Two additional studies carried out in the USA and Italy supported these findings by reporting a prevalence of DQ2/8 negativity in $\mathrm{CD}$ ranging from $0 \cdot 16$ to $0.9 \%^{(11,12)}$. When clinical suspicions are strong and supported by serological and histological findings in small samples of patients, CD can be diagnosed in the absence of HLA-DQ2 or -DQ8. Recently, genome-wide association studies have been able to identify several common nonHLA genetic factors associated with CD that account for a small amount of the overall risk. These genes have been reported to also be involved in adaptive and innate immune responses.

It should be noted that each of these non-HLA genes, taken separately, is not expected to play a major role in $\mathrm{CD}^{(6,7)}$. A study has also reported an association between HLA-G gene polymorphisms and susceptibility to CD development, suggesting the involvement of the HLA-G molecule in the pathogenesis of the disease ${ }^{(13)}$.

\section{Environment}

It is clear that environmental factors play an important role in CD pathogenesis. The major environmental trigger is the intake of gluten. The problem lies in the high content of glutamine and proline concentrated in these proteins that make them impossible to be completely digested by humans ${ }^{(6)}$. Individuals genetically predisposed to CD undergo a process by which the partially residual digested peptides set off an innate and adaptive immune response, thus considering a GFD to be the only effective treatment for $\mathrm{CD}$ to date. There may be other trigger factors in the development of the disease that could account for the considerable variability in the age of onset and in the clinical manifestations of the disease. Current studies have focused on these other factors such as the gut microbiome and its role in contributing to disease onset ${ }^{(14)}$, or the lack of breastfeeding ${ }^{(15)}$. As far as the latter is concerned, several researchers have identified a relationship between breast-feeding and the development of $\mathrm{CD}^{(15,16)}$. Both the duration of breastfeeding and the delay until gluten is introduced have been associated with a decrease in the risk of developing CD, though its cause still remains unknown. Nevertheless, other studies do not support this hypothesis. Indeed, A study of Italian children with a familial risk of $\mathrm{CD}^{(17)}$ showed that neither the delayed introduction of gluten nor breast-feeding modified the risk of CD development among infants at risk, although the introduction of gluten at a later time is associated with a delayed initiation of the disease. Another study showed no effect of the timing of gluten being introduced on the incidence of CD in children at risk ${ }^{(18)}$

Furthermore, studies report differences in the microbiota of breast-fed and formula-fed infants ${ }^{(19)}$. It has been reported that the microbiota, as well as nutritional and immune systemsupporting factors in breast milk, could contribute to a decrease in gastrointestinal illnesses. Interestingly, infections by a variety of pathogens (for example, adenovirus 12 and hepatitis $\mathrm{C}$ virus $(\mathrm{HCV})$ ), have been associated with $\mathrm{CD}^{(20)}$. Moreover, the risk rate for $\mathrm{CD}$ diagnosis and the age of onset seemed to increase in children with CD-associated HLA genes after one or more rotavirus infections ${ }^{(21,22)}$. In addition, epidemiological studies report that individuals with $\mathrm{CD}$ were likely to be born during the summer period, meaning that the introduction of solid food, which occurs around 6 months of age, is concurrent with the seasonal peak of gastrointestinal illnesses in the winter period $^{(23,24)}$. There is no clear evidence of a direct relationship between infections and the onset of $\mathrm{CD}$, but it has been suggested that rotaviruses and other intestinal pathogens can create a pro-inflammatory environment and increase intestinal permeability ${ }^{(25)}$, thus enhancing the immune response to dietary antigens. In response to viral infections, there is a production of interferon (IFN)- $\alpha$ that enhances the activation of the Th1 response to anti-CD3 in the small intestine, leading to an increase in crypt hyperplasia ${ }^{(26)}$. Studies report numerous cases of patients with $\mathrm{HCV}$ who, after being treated with IFN- $\alpha$ therapy, developed $\mathrm{CD}$, thus supporting a possible role played by type I IFN in the induction of the disease ${ }^{(27,28)}$.

\section{Immunopathogenesis}

Immune dysregulation has been the core feature examined over the last few decades. Before addressing the issue of immunopathogenesis, an explanation of the amino acid composition of gluten is necessary. Gluten is comprised of two different protein types, gliadins and glutenins, which trigger the disease ${ }^{(29,30)}$. 
Peptides in barley and rye, hordeins and secalins are also capable of activating CD while oats, with its more distantly related peptides called avenins, rarely trigger $\mathrm{CD}^{(31)}$. Due to their high content of prolines and glutamines, gliadins, glutenins, hordein and secalins are resistant to the degradation of gastric acid, pancreatic and brush-border enzymes, due to the lack in prolyl endopeptidase activity ${ }^{(32)}$. Undegraded peptide fragments can be transported across the epithelium primarily by transcellular pathways, such as tight junctions, which contribute to the balance between tolerance and immune responses to non-self antigens ${ }^{(33)}$. Zonulin, an endogenous modulator of epithelial tight junctions, triggers the paracellular trafficking of macromolecules. An increase in the secretion of zonulin is produced by gliadin, altering intestinal permeability and facilitating the transport of gluten, and triggering an inflammatory process. At the initial stage of the disease, exposure to gliadin induces directly the opening of tight junctions secondary to zonulin up-regulation and produces an increase in the paracellular passage of antigens in the gut submucosa ${ }^{(34)}$. Peptide fragments from wheat, rye and barley that are not digested are transported to the lamina propria, where they undergo the process of deamidation by tTG2 resulting in the conversion of glutamine into glutamate which in turn introduces negative charges with a stronger binding affinity for HLA-DQ2 and -DQ8 on antigen-presenting cells ${ }^{(35,36)}$. It is worth noting that gluten peptides that can be modified through deamidation are numerous, thus broadening and amplifying the gluten-specific T-cell response in the lamina propria ${ }^{(37)}$. It has been demonstrated that $\mathrm{CD}^{+} \mathrm{T}$ cells produce most of the $\mathrm{CD}$ features ${ }^{(38)}$, recognising the gluten peptides bound to HLADQ2.5 Or HLA-DQ8 ${ }^{(39)}$, and amplifying the T-cell response through tTG2. Bodd et al. ${ }^{(40)}$ have reported that $\mathrm{T}$ cells reacting against translutaminase deamidated gliadin are IFN- $\gamma$-secreting cells. IFN- $\gamma$ induces the production of matrix metalloproteases $^{(41,42)}$ leading to the alteration of the epithelial barrier function $^{(3)}$. Moreover, both IFN- $\gamma$ and TNF- $\alpha$ are reported to increase intestinal permeability by the disruption of tight junctions $^{(43)}$. On the other hand, antigen-presenting cells generate IL-12 and IL-15, which are responsible for the promotion of the Th1 differentiation, survival and proliferation of gluten-specific $\mathrm{CD}^{+}{ }^{+} \mathrm{T}$ cells ${ }^{(44)}$. $\mathrm{CD}^{+} \mathrm{T}$ cells respond with the secretion of proinflammatory cytokines controlling local inflammation, such as IFN- $\gamma$, IL-2 and IL-21 $1^{(40,45)}$. These cytokines act not only at a local level, but they also cross the basement membrane and bind receptors on the intestinal epithelial cells (IEC) and intraepithelial lymphocytes (IEL) ${ }^{(7)}$. In the epithelium, IFN- $\gamma$ promotes IEC death and possibly IL- 15 production by IEC $^{(46)}$. In vitro studies have shown that IL-15 can activate T-cell receptor $\alpha \beta$ (TCR $\alpha \beta)$ IEL, leading to an increase in surface levels and activity of NKG2D (natural killer (NK) group 2D), the receptor for MHC class I polypeptide-related sequence A (MICA) ${ }^{(47)}$ which can be found on the membrane of the CD8 ${ }^{+}$ $\alpha \beta$ T cells, $\gamma \delta$ T cells and most NK cells ${ }^{(48)}$, resulting in death. It is noted that CD patients who suffer from CD have an increase in both the expression and function of NKG2D in TCR $\alpha \beta$ IEL $^{(49)}$. It is suggested that, following NKG2D-MICA interaction, the TCR $\alpha \beta$ IEL become activated, and kill the IEC, thus contributing to epithelial pathology because the IEL are reprogrammed to become NK cells ${ }^{(48)}$. Furthermore, it has been shown that the expression of epithelial cell surface ligands, including MICA, is increased by IL-15, thus contributing to epithelial changes and other pathological processes associated with $\mathrm{CD}$, which include the refractory $\mathrm{CD}$ type 2 and enteropathy associated T-cell lymphoma ${ }^{(50,51)}$. The interaction between IL-15 and the $\mathrm{CD}^{+}$ $\mathrm{T}$ cells is a necessary and sufficient condition to activate the $\mathrm{CD}^{+} \mathrm{T}$ cells and damage the small intestine ${ }^{(38)}$. Usually acting as an autocrine growth factor, IL-21 has shown to have several functions. In the epithelium, the IEC are stimulated to produce CCL20, a T-cell chemoattractant, and enhance the cytotoxicity of the IEL. In the lamina propria, it is associated with the production of matrix metalloproteases by fibroblasts ${ }^{(52)}$, supporting the growth and differentiation of B cells. IL-21 also supports B cells in the production of IgA antibodies, specific for TG2 or deamidated gliadin ${ }^{(53)}$. Finally, IEC produce IL-7 and IL-15 which promote the activation and survival of IEL $^{(54)}$. The activated IEL, in turn, have the ability to kill IEC. Furthermore, the activation and clonal expansion of $\mathrm{B}$ cells is driven by the activated $\mathrm{CD}^{+} \mathrm{T}$ cells, through the production of Th-2 cytokines, so that $\mathrm{B}$ cells differentiate into plasma cells and produce antigliadin and anti-tTG antibodies ${ }^{(55)}$. These processes are thought to contribute to several features of $\mathrm{CD}$ pathology such as increased IEL numbers, villous atrophy, land the production of disease-specific antibodies, with the effect of producing inflammation, malabsorption and numerous secondary symptoms ${ }^{(56)}$.

\section{Neurological complications and cognitive impairment in coeliac disease}

CD is primarily an intestinal disorder characterised histologically by intraepithelial lymphocytosis, crypt hyperplasia and villous atrophy. This broader view of the disease seen as an inflammatory disease is supported by clinical observations of extra-intestinal manifestations such as dermatological, hepatic, osteological, endocrine and neurological signs ${ }^{(57)}$. The most severe neurological symptoms are dementia, amnesia, ataxia, acalculia, epilepsy, chronic neuropathies, confusion, personality changes ${ }^{(58)}$, cognitive deficits ${ }^{(59)}$, multifocal encephalopathy, neuromyelitis optica, muscular hypotonia, delayed motor development ${ }^{(60)}$ and headaches ${ }^{(61,62)}$, some of which could improve with GFD treatment ${ }^{(61,63)}$.

Due to high levels of antigliadin antibodies, CD is seen as the common cause of neurological syndromes, particularly cerebellar ataxia, whose origin remains unknown. There is an increasingly wider spectrum of neurological syndromes identified both as complications of prediagnosed CD and as an initial manifestation of $\mathrm{CD}^{(64)}$. Studies show that the nervous system is compromised and acts as a complication in the pre-diagnosis of CD. Many studies have reported the involvement of the nervous system mainly as a complication of prediagnosed $\mathrm{CD}^{(61,65)}$. Several studies have investigated the prevalence of neuronal hyper-excitability and electroencephalogram (EEG) abnormalities in asymptomatic children and adolescents with newly diagnosed CD before the introduction of a GFD, and in particular any changes following the introduction of a diet. 
A recent study evaluated the role of a GFD on neurological symptoms, EEG characteristics and sleep breathing disorders (SBD) in children with $\mathrm{CD}^{(61)}$. The authors enrolled nineteen children with a new biopsy $\mathrm{CD}$ diagnosis. At the diagnosis of $\mathrm{CD}, 37 \%$ of the patients complained of headaches and other neurological disorders that affected their daily activities and $32 \%$ of the patients were positive for SBD. The EEG tests revealed abnormal findings in $48 \%$ of children. After 6 months of a GFD, headaches and EEG abnormalities disappeared in most of the children in turn, resulting in them being negative for SBD. Asymptomatic children and adolescents manifested hyper-excitability and EEG abnormalities before a GFD. Adherence to a GFD has been shown to result in lighter headache symptoms reported by CD patients ${ }^{(62)}$. Results of functional imaging studies such as single photon emission computed tomography were in favour of migraines, and a GFD could lead to an improvement of migraine in these patients ${ }^{(66)}$. Functional imaging studies revealed migraines present through single photon computed tomography with the result that a GFD could lead to an improvement. However, an association between CD and migraine headaches was not established when compared with the general population ${ }^{(67)}$.

Other common neurological symptoms of CD are ataxia and epilepsy. Cerebellar ataxia is one of the first symptoms ${ }^{(68)}$, and one of the most frequently recognised neurological disturbances in $\mathrm{CD}^{(69)}$. Its predominant clinical manifestations include dysarthria, dysphonia, pyramidal signs, abnormal movements of eyes and progressive ataxia of gait. Ataxia related to $\mathrm{CD}$ is not often associated with typical gastrointestinal symptoms or malabsorption signs. Ataxic symptom relief has been reported with the adherence to a GFD ${ }^{(70)}$. Regarding epilepsy, several studies have demonstrated a relationship between epilepsy and $\mathrm{CD}$, with prevalence rates ranging from $3 \cdot 5$ to $7 \cdot 2 \%^{(64,69)}$. However, although epilepsy is considered in international studies as a condition associated with CD or an extra-intestinal manifestation of this disease, a recent study has not confirmed this association ${ }^{(60)}$.

Recent data are less consistent and vary below these figures $^{(71)}$. Peripheral neuropathy is the second most frequent neurological complication in CD patients and has been reported in up to $50 \%$ of cases ${ }^{(61,72)}$. Neuropathy may precede a diagnosis of $\mathrm{CD}^{(73)}$. Research findings regarding the effect of a GFD on peripheral neuropathy have been mixed, with some reports indicating positive outcomes and others reporting no significant changes ${ }^{(63,74)}$.

A study which investigated neurological damage and glutenfree foods showed that neurological peripheral disorders, and autonomic dysfunctions with anti-neuronal antibodies in adults with $\mathrm{CD}$ did not disappear with a GFD ${ }^{(75)}$. On the contrary, important evidence comes from a case report of a 14-year-old patient with rare neurological symptoms ${ }^{(76)}$. The patient presented headaches and blurred vision for 1 year; she was diagnosed with papilledaema after an ophthalmic examination showing increased cerebrospinal fluid putting pressure on the backbone and was further diagnosed with pseudotumor cerebri. However, upper gastrointestinal endoscopy with duodenal biopsy confirmed the diagnosis of $\mathrm{CD}$. The patient was put on a GFD, resolving the gastrointestinal symptoms and also reaching almost complete resolution of the pseudotumour cerebri symptoms.

Neurological signs are rare in children but as many as $36 \%$ of adult patients present neurological changes ${ }^{(77)}$. Other neurological manifestations are tremor, myelopathy, brainstem encephalitis, progressive leukoencephalopathy, vasculitis, occipital calcification and myoclonic syndrome. A myoclonic syndrome, often accompanied by ataxia, may occur in CD. Myoclonus may be present as focal, multifocal or generalised convulsions. They also include neuromuscular manifestation such as peripheral polyneuropathy, mono-neuropathy multiplex, dermatomyositis, polymyositis and inclusion body myositis ${ }^{(67)}$. Additionally, one study showed that there is no significant difference between the laboratory data of the coeliac patients with and without neurological manifestations ${ }^{(78)}$. The neurological symptoms are headaches, epilepsy, migraines, mental retardation, breathholding spells, ataxia, cerebral palsy, attention-deficit/hyperactivity disorder, Down's syndrome and Turner syndrome in order of frequency. Moreover, the 3a biopsy type was statistically more common among patients without neurological manifestations, while the 3b biopsy type was statistically and more common among patients with neurological manifestations. It is important to keep in mind that in the clinical course of $\mathrm{CD}$, different neurological manifestations may be reported.

Although a range of neurological disorders are widely reported to be associated with coeliac patients, their pathogenesis remains unclear. Such disorders are believed to be secondary to vitamin deficiency due to malabsorption, and others to immune mechanisms. A further detailed study confirmed the role of hyperhomocysteinaemia for neurological features associated with $\mathrm{CD}^{(79)}$.

Neurological conditions have been researched in detail as opposed to cognitive functioning. CD adult patients reported milder forms of cognitive impairment known as 'brain fog', a symptom which disappears after GFD treatment but re-presents itself again after inadvertent gluten exposure ${ }^{(80)}$. Brain fog is characterised by the difficulty in concentrating, problems with attentiveness, short-term memory lapses, difficulties in wordfinding, temporary loss in mental acuity and creativity as well as confusion and/or disorientation ${ }^{(81)}$.

A retrospective study on patients with a CD diagnosis after the age of 60 years presented evidence supporting the likelihood of such a link. Two patients out of seven presented a cognitive decline, initially attributed to Alzheimer's disease, but they showed an improvement in their symptoms after the initiation of a GFD. A third of the patients suffered from a peripheral neuropathy, and completely recovered following a gluten-free regimen ${ }^{(82)}$. The level of cognitive function was believed to have improved together with mucosal healing, an hypothesis proposed by Lichtwark et al. ${ }^{(80)}$ in their longitudinal pilot study which investigated the relationships between cognitive function and mucosal healing in individuals diagnosed with CD starting a GFD. The study tested eleven patients (eight females, three males), mean age 30 (range 22-39) years, on a battery of cognitive tests at 0,12 and 52 weeks. The items under examination were information, processing efficacy, memory, visual-spatial ability, motoric function and attention. Researchers also collected small bowel 
biopsies via routine gastroscopy at 12 and 52 weeks so that cognitive performance could be compared with serum concentrations of tTG antibodies, with biopsy outcomes and with other biological markers. The results of the paper showed that tTG antibody concentrations decreased from a mean of 58.4 at baseline to $16.8 \mathrm{U} / \mathrm{ml}$ at the $52 \mathrm{nd}$ week, while four of the cognitive tests assessing verbal fluency, attention and motoric function reported significant improvement over the 12 weeks, besides confirming that improvement in cognitive performance was parallel to mucosal healing. The study reported an excellent adherence to the diet by all patients.

In addition, a study by Casella et al. ${ }^{(83)}$ evaluated the functional and cognitive performance in CD patients diagnosed at the age of 65 years or older, compared with age- and sexmatched control patients using psychometric tests to obtain comprehensive information on functional performance, and general or more specific cognitive functions. The results evidenced how cognitive performance is worse in the elderly than in control patients, despite a longer time on a GFD ${ }^{(83)}$.

Several studies have investigated the relationship between dementia and $\mathrm{CD}^{(59)}$. Dementia develops as acalculia, confusion, amnesia and personality disorder ${ }^{(82)}$. However, the aetiology and specific treatment of this complication are not clearly known ${ }^{(67)}$. Collin et al $^{(84)}$ reported five cases of patients with $\mathrm{CD}$ who developed dementia before the age of 60 years. Four patients suffered intellectual deterioration ranging from moderate to severe. In addition to intellectual deterioration, one patient showed deficit in both verbal and visual memory. One had slow psychomotor functions, and the other showed severe memory deficit, constructional deficit and apraxia. The fifth patient had deficits in digit span, visual memory, and visualmotor constructional difficulties. Brain computed tomography revealed diffused cerebral or cerebellar atrophy in all patients ${ }^{(84)}$. Other studies have reported a link between dementia in individuals diagnosed with $\mathrm{CD}$ after the age of 60 years. A retrospective study in Israel examined seven patients diagnosed with $\mathrm{CD}$ after the age of 60 years. Of the seven, two female patients were initially diagnosed with dementia of the Alzheimer type due to a progressive cognitive decline. Compliance to a GFD ameliorated the cognitive decline in both patients ${ }^{(82)}$. However, in another study, adherence to a GFD resulted in no change in neurological symptoms in patients with CD who presented mild memory impairment ${ }^{(85)}$.

\section{Psychiatric disorders and quality of life of coeliac disease patients}

The association of $\mathrm{CD}$ with psychiatric disorders, including depression, has been identified for a long time. Several psychiatric symptoms have been reported as common complications in many patients suffering from $\mathrm{CD}$, though the effects of diet on one's mood and psychiatric symptoms remain largely unknown ${ }^{(86)}$. Psychiatric symptoms usually described in CD patients include depressive symptoms, apathy, excessive anxiety, irritability ${ }^{(65)}$, eating disorders ${ }^{(87)}$, attention-deficit/ hyperactivity disorder ${ }^{(88)}$ and autism $^{(89)}$ as well as sleep disorders which are also common in CD. Sleep disorders are related to depression, anxiety and fatigue, and inversely related to $\mathrm{QOL}^{(90)}$.

Carta et $a l .{ }^{(91)}$ suggests that the possible causative factors are malabsorption and nutritional deficiencies (especially of vitamin $\mathrm{B}_{6}$ and tryptophan) and the association with other autoimmune diseases such as thyroid disease. A study on untreated CD patients reported decreased plasma levels of tryptophan and other monoamine precursors and decreased cerebrospinal fluid levels of serotonin, dopamine and noradrenaline metabolites (5-hydroxyindoleacetic acid, homovanillic acid and 3-methoxy-4-hydroxyphenylglycol, respectively) ${ }^{(92)}$. A cross-sectional, case-control study enrolling thirty-six CD patients used the Composite International Diagnostic Interview to assess lifetime psychopathology. The findings showed that the risks of major depression $(41.7 \%)$, dysthymic disorder (8.3\%), adjustment disorders (30.5\%) and panic disorder (13.9\%) are higher in $\mathrm{CD}^{(91)}$.

A meta-analysis on anxiety and depression in CD found that depression is more common and more severe in adults with CD compared with normal controls. However, depression in adults with CD did not differ from adults who had other physical illnesses and no differences in anxiety were found ${ }^{(93)}$. Additional studies have also found increased rates of depressive and anxiety symptoms in $\mathrm{CD}$ patients on a $\mathrm{GFD}^{(94,95)}$, though the prevalence of depressive symptoms in $\mathrm{CD}$ patients varies widely across studies ranging from 6 to $69 \%$ as shown by another large longitudinal population-based cohort study ${ }^{(96)}$, where $\mathrm{CD}$ patients were also reported to have an $80 \%$ increased risk of depression compared with controls. This variability in prevalence could be accounted for by differences in personal characteristics, cultural background and study design. The increased risk of depressive symptoms in CD patients could be explained by several mechanisms.

The first mechanism could be dietary non-compliance and sustained malabsorption, which could lead to sustained nutritional deficiencies (for example, vitamin $\mathrm{B}_{6}$, vitamin $\mathrm{B}_{12}$ and folic acid) producing the risk of depression ${ }^{(97)}$. Second, a restrictive GFD could cause nutritional deficiencies. Even though these nutritional deficiencies contribute to the risks of depression, it is not established to date ${ }^{(98)}$. Depression could also be induced by reductions in brain monoamine availability and metabolism ${ }^{(92)}$ and regional cerebral hypoperfusion ${ }^{(99)}$ in patients suffering from CD. Delgado et al. ${ }^{(100)}$ suggested excessive cytokine secretion due to chronic immune system activation as a fundamental pathology underlying depressive symptoms. Furthermore, there is an increased hypothalamicpituitary-adrenal axis hyperactivity due to the cytokine activation associated with major depression ${ }^{(101)}$. Another reason can be found in the fact that, due to the strict dietary regimen, CD patients may avoid social situations involving food experiences. They may have higher levels of psychological distress because of daily difficulties, negative responses to the diet due to social surroundings and continuous worrying about dietary mistakes and negative results which could all lead to depression $^{(102)}$. Finally, depression in CD patients may be a secondary condition resulting from the association between CD and other auto-immune diseases with a high risk of depression, such as thyroid disease and diabetes mellitus ${ }^{(103)}$. 
Relationships between CD and psychiatric disorders such as anxiety and depression have been described especially when CD starts after 60 years of age ${ }^{(67)}$. It has been shown that children and adolescents with CD may have emotional and behavioural problems ${ }^{(104)}$. Most CD patients showed significantly higher scores of anxiety, harm avoidance, separation panic and somatic complaints, even after the introduction of dietary regimens. The introduction of a GFD results in a radical change in eating habits and lifestyle of CD children, and can be difficult to accept and stressful to follow. This induces a high level of anxiety, which may be evidenced in a different way according to gender susceptibility, resulting in depression in females and aggression and irritability in males. The acceptance of a GFD also depends on age. For example, adolescents aged between 12 and 17 years find it particularly difficult to adapt to their new dietary regimen. This is due to the fact that this age bracket already has to manage with social interactions with their peers and adults. Overall, reports highlight the fact of having a chronic condition during childhood and adolescence could prove difficult to manage. In this context, a strict food regimen can be considered a negative influence on their social life. On the whole, all these reports indicate that the impact of a chronic condition during childhood and adolescence may be difficult to manage ${ }^{(104)}$. On the other hand, another study demonstrated an association of increased depression and disruptive behavioural disorders in adolescents with untreated $\mathrm{CD}$. This is evidence supporting the improvement in psychiatric symptoms after the initiation of a GFD ${ }^{(105)}$. A case-report also suggests that CD should be taken into consideration in children with psychiatric disorders, particularly if they are not responsive to psychotropic medication ${ }^{(106)}$. Given that unrecognised CD may predispose the sufferer to serious psychiatric disorders and behavioural problems, it should be considered as a differential diagnosis in all age groups. A recent study aimed to establish whether long-term adherence to a GFD can be related to depressive symptoms in $\mathrm{CD}$ patients, given that lifetime depressive symptoms may be present in at least one-third of CD patients on a GFD. The results of a recent study on long-term adherence to a GFD ( 5 years) evidenced a decrease in depressive symptoms producing relief of the symptoms as opposed to a short-term diet of less than 2 years ${ }^{(107)}$. It is worth noting that the elimination of gluten in the case of a patient suffering from chronic treatment-resistant symptoms of depression and anxiety showed significant improvement in the mental state and in routine activities. When anxiety and depressive symptoms persist even after an unsatisfactory reaction to pharmacological treatment, it indicates the need to identify the somatic reasons for the underlying condition $^{(108)}$. Interestingly, a reduced QOL was highlighted in $\mathrm{CD}$ patients as compared with healthy control participants $^{(109)}$.

Recent epidemiological data highlight the association between schizophrenia (SCZ) and several autoimmune diseases $^{(110,111)}$, CD being one found to be in association with SCZ since the $1950 \mathrm{~s}^{(112)}$. Studies on the effects of the elimination of gluten from the diet of SCZ patients have further strengthened the existence of an association between gluten and SCZ. In fact, SCZ patients whose symptoms improved after the introduction of a cereal- and milk-free diet showed an interruption or reversal of clinical improvement towards wheat ${ }^{(113,114)}$. A few studies have suggested that SCZ and CD may be associated with similar or adjacent genes ${ }^{(115,116)}$. It has been reported that genetic susceptibility in SCZ lies in the HLA DQ, similarly to autoimmune disorders such as $\mathrm{CD}^{(117)}$. By contrast, a recent study showed no such HLA association in $\mathrm{SCZ}^{(118)}$.

The subsequent observations provided contrasting results. Epidemiological studies, for example the National Danish Register ${ }^{(119)}$, found that CD occurred before the onset of SCZ and that antibody-based diagnosis (above all anti-gliadin) was a risk factor for $S C Z^{(118,120)}$. A study observed that anti-gliadin antibodies have a role in the aetiopathogenesis of $\mathrm{SCZ}^{(118)}$. An increasing number of studies suggest that the immune mechanisms are partially responsible for SCZ. Similarly to $\mathrm{CD}$, an aberrant Th1 immune reaction has been connected with the development of SCZ. The study suggests a causal link between CD and SCZ: the risk for SCZ in $\mathrm{CD}$ patients may be dependent on the interplay between IFN- $\boldsymbol{\gamma}$ and the transcription factor STAT1 (signal transducer and activator 1$)^{(121)}$.

A recent study showed that bacterial compositions could explain the inefficient gluten digestion and how in certain situations can be a risk factor for SCZ because the gut microbiota contributes to digestion, inflammation, gut permeability and behaviour ${ }^{(122)}$. It is also significantly recognised that bidirectional communication exists between the brain and the gut that uses neural, hormonal and immunological routes. An increased incidence of gastrointestinal barrier dysfunction, food antigen sensitivity, inflammation and the metabolic syndrome is seen in $\mathrm{SCZ}{ }^{(123)}$. As demonstrated, these symptoms can be influenced by the composition of the gut microbiota.

Another theoretical framework suggests that inappropriate oestrogen exposure occurring in the brain could also be occurring in the colon so that an association of $\mathrm{CD}$ or some other inflammation and SCZ may be observable not from a genetic link, but rather from a transgenerational effect of prenatal oestrogen exposure ${ }^{(120)}$.

There have been studies and case reports of a dramatic recovery from SCZ associated with the implementation of a GFD. A case report shows that there is a improvement in psychotic symptoms after a GFD in a young man with complex autoimmune illness ${ }^{(124)}$. The remission of psychotic symptoms in this patient was associated with the adherence to the diet. Regardless of the exact mechanism involved, the marked improvement in this patient's SCZ symptoms after the implementation of a GFD highlights the need for further research on the role of the diet in SCZ.

Another research group reported a case of brain perfusion abnormalities, assessed by single photon emission computed tomography examination, in a 33-year-old CD patient with SCZ, with the regression of both cerebral hypoperfusion and SCZ symptoms observed after 6 months of a $\mathrm{GFD}^{(125)}$. These findings may have potential implications for the treatment of these subjects given that a GFD can contribute to the improvement of their symptoms. In another case report, the symptoms of SCZ were improved in a coeliac patient after the introduction of a GFD ${ }^{(126)}$. 
To date, CD adversely affects the QOL of individuals because of several factors such as its chronic nature, the impact on health, psychological distress, social and family connotations, and the need for lifelong treatment. While these factors are important for QOL, one area that has received less attention are the psychological symptoms and how to cope, despite the higher rates of psychological symptoms within CD patients compared with the general population.

There is proven evidence showing that when one suffers from CD it affects the general perception of their QOL and wellbeing and a GFD generates difficulties and limitations in the life of patients under treatment ${ }^{(127)}$. Even in the case of a considerable improvement in the symptoms, adhering to a GFD can be difficult for many individuals because of poor palatability and poor availability of GF products, thus resulting in a condition that can have serious repercussions on the $\mathrm{QOL}^{(128)}$. Casellas et al. ${ }^{(129)}$ have recently reported that good adherence to a GFD resulted in an improved QOL using CD-QOL compared with patients with the intention of not complying. It could be affirmed that it is only with a complete adherence to a GFD that brings significant benefits for the health and QOL of coeliac patients in order that its drawbacks can be counterbalanced. In conclusion, the results of this study suggest that most coeliac patients who followed a GFD correctly showed a better QOL, measured by a specific instrument for coeliac patients and this was also associated with a good control of the symptoms ${ }^{(129)}$. It has been demonstrated that there is a direct link between the severity of gastrointestinal and psychological symptoms in coeliac patients and how their symptoms both have an impact on $\mathrm{QOL}^{(130)}$. This research is the first which investigates the medical impact of the disease and its psychological effects and how adherence to a GFD has effects on the QOL in CD. This study shows how poor adherence to a GFD reduces the QOL which in turn increases the psychological symptoms as well as severe gastrointestinal symptoms and difficulty in coping. These results represent major threats in achieving an adequate QOL. Early diagnosis and treatment of this disease could alleviate the symptoms which in turn could lead to a better QOL.

Potential link between the intestine and the brain: the role of a gluten-free diet

Mental disorders that accompany digestive diseases constitute an interdisciplinary aspect to date not fully recognised and considered a diagnostic and therapeutic problem. The most recognised is CD in which patients suffer from a wide range of neuropsychiatric symptoms. It has not been fully explained how the pathogenic mechanism of $\mathrm{CD}$ affects the patient's mental health, but one hypothesis suggests that it is due to serotonin imbalance or opioid neurotransmission caused by the effect of gluten and gluten metabolites on the central nervous system $(\mathrm{CNS})^{(131)}$.

Given that the gastrointestinal tract is connected to the CNS means that the communication involves neural pathways and immune and endocrine mechanisms. The intestinal barrier prevents toxins, pathogens and antigens in altering the various neuroactive compounds ${ }^{(132)}$.
The existence of a rich gut-to-brain communication raises the possibility that intestinal barrier alterations may take part in the pathophysiology of CNS disorders and determine neuropsychiatric symptoms ${ }^{(133)}$. To date there have been neurophysiological studies aiming to evaluate the gut-microbiota-brain axis ${ }^{(134,135)}$ but these unfortunately have not yet involved coeliac patients. A study suggests that modulation of the gut microbiota may provide a novel therapeutic target for the treatment and/or prevention of mood and anxiety disorders ${ }^{(136)}$. Alterations in gut microbial composition is associated with marked changes in behaviours relevant to mood, anxiety and cognition, establishing the critical importance of the bi-directional pathway of communication between the microbiota and the brain in health and disease. Dysfunction of the gut-microbiota-brain axis has been implicated in stress-related disorders such as depression, anxiety and neurodevelopmental disorders such as autism and $\mathrm{SCZ}^{(137)}$.

Although communication between gut microbiota and the CNS are not fully elucidated, neural, hormonal, immune and metabolic pathways have been suggested. The concept of a gutmicrobiota-brain axis is emerging, suggesting that microbiotamodulating strategies may be a tractable therapeutic approach for developing new treatments for CNS disorders and for neurological and psychiatric symptoms ${ }^{(138)}$. Several studies have reported the effect of a GFD on the composition of the gut microbiome in CD patients ${ }^{(139,140)}$, Patients with $\mathrm{CD}$ have a reduction in beneficial species and an increase in those potentially pathogenic as compared with healthy subjects ${ }^{(141)}$. Gut microbes can produce hormones and neurotransmitters and the bacterial receptors for these hormones influence microbial growth and pathogenicity. Gut bacteria directly stimulate afferent neurons of the enteric nervous system, sending signals to the brain via the vagus nerve ${ }^{(142)}$. Through these varied mechanisms, gut microbes induced reactivity of the hypothalamic-pituitary-adrenal axis which influence memory, mood and cognition and are clinically and therapeutically relevant to a range of disorders. In order to alter the gut microbiome therapeutically, changes in diet, probiotics and prebiotics are necessary ${ }^{(143)}$. Some reports show that in autism disorders, the use of diets such as gluten-free and casein-free diets may contribute to the improvement in behavioural symptoms following these dietary regimens ${ }^{(133)}$. In patients under a GFD, a global increase in cortical excitability was observed in one study, suggesting a glutamate-mediated functional reorganisation compensating for the progression of the disease ${ }^{(144)}$. It was hypothesised that glutamate receptor activation, probably triggered by $\mathrm{CD}$ related to immune system dysregulation, might result in a long-lasting motor cortex hyperexcitability with increased excitatory post-synaptic potentials, probably related to the phenomena of long-term plasticity. In this study, a certain degree of improvement of depressive symptoms was also observed, supporting the role of a GFD in the amelioration of psychiatric CD-related disorders. Another study reports that immune system dysregulation in patients with $\mathrm{CD}$ may play a central role in triggering changes in motor cortex excitability resulting in the manifestation of the symptoms ${ }^{(145)}$. This study suggested that cross-reaction between anti-gliadin antibodies and neuronal antigens, as well as altered levels of ions related to transglutaminase deposition 6-immunoglobulin, could affect the normal balance between excitatory and inhibitory 
synaptic excitability. To emphasise the importance of these links, psychiatric or neurological patients may benefit from starting a GFD stressing the importance that there is a two-way communication between the brain and the gut that uses neural, hormonal and immunological pathways. In the future, scientific researches could prove that there is a link between the intestine and the brain, and that GFD could exert a role for the treatment of neuropsychiatric symptoms.

\section{Conclusions}

Based on current evidence, the present review proposes a comprehensive view of the pathogenesis of $\mathrm{CD}$ and explains the progression of its development and of its complications. From a genetic basis, the immunopathogenesis and above all the neurological or psychiatric implications of recent scientific studies where $\mathrm{CD}$ is becoming more complicated is discussed. The purpose of the present review was to investigate and illustrate the neurological and psychiatric complications in CD and the importance of a GFD given the symptoms. While some of these symptoms can improve with a GFD, our advice is to try to diagnose $\mathrm{CD}$ as early as possible, given that delays in the diagnosis may cause severe implications in the nervous system. The importance of early diagnosis is fundamental and the only treatment available is a GFD to be followed for a lifetime. It is therefore essential to follow nutritional therapy to avoid this kind of complication. Mental disorders accompanying digestive system diseases are interdisciplinary although poorly acknowledged. One of the most recognised examples is $C D$ in which patients suffer from a wide range of psychopathological symptoms, which must be taken into consideration from both a diagnostic and therapeutic point of view. The above studies show that neuropsychiatric symptoms may represent an atypical manifestation of $\mathrm{CD}$ occurring before the gastroenterological diagnosis and the introduction of the diet causes a significant improvement in mental status. Bearing in mind these considerations, our review also claims that a GFD is effective in the treatment of depression, anxiety and neurological complications associated with $\mathrm{CD}^{(105,107)}$.

\section{Acknowledgements}

The authors thank Ms Filomena Natarelli for the editing of the manuscript.

The present review received no grant from sponsors or from commercial or non-profit organisations.

There are no conflicts of interest.

\section{References}

1. Husby S, Koletzko S, Korponay-Szabó IR, et al. (2012) European Society for Pediatric Gastroenterology, Hepatology, and Nutrition guidelines for the diagnosis of coeliac disease. $J$ Pediatr Gastroenterol Nutr 54, 136-160.

2. Catassi C (2014) The new epidemiology of celiac disease. J Pediatr Gastroenterol Nutr 59, Suppl. 1, S7-S9.

3. Miranda J, Lasa A, Bustamante MA, et al. (2014) Nutritional differences between a gluten-free diet and a diet containing equivalent products with gluten. Plant Foods Hum Nutr 69 , 182-187.
4. Castillo NE, Theethira TG \& Leffler DA (2015) The present and the future in the diagnosis and management of celiac disease. Gastroenterol Rep 3, 3-11.

5. Di Sabatino A \& Corazza GR (2009) Coeliac disease. Lancet 373, 1480-1493.

6. Kupfer SS \& Jabri B (2012) Celiac disease pathophysiology. Gastrointest Endosc Clin N Am 22, 639-660.

7. Denham JM \& Hill ID (2013) Celiac disease and autoimmunity: review and controversies. Curr Allergy Asthma Rep 13, 347-353.

8. Kim CY, Quarsten H, Bergseng E, et al. (2004) Structural basis for HLA-DQ2-mediated presentation of gluten epitopes in celiac disease. Proc Natl Acad Sci U S A 101, 4175-4179.

9. Hovhannisyan Z, Weiss A, Martin A, et al. (2008) The role of HLA-DQ8 $\beta 57$ polymorphism in the anti-gluten T-cell response in coeliac disease. Nature 456, 534-538.

10. Karell K, Louka AS, Moodie SJ, et al. (2003) HLA types in celiac disease patients not carrying the $D Q A 1 * 05-D Q B 1 * 02$ (DQ2) heterodimer: results from the European Genetics Cluster on Celiac Disease. Hum Immunol 64, 469-477.

11. Megiorni F, Mora B, Bonamico M, et al. (2009) HLA-DQ and risk gradient for celiac disease. Hum Immunol 70, 55-59.

12. Pietzak MM, Schofield TC, McGinniss MJ, et al. (2009) Stratifying risk for celiac disease in a large at-risk United States population by using HLA alleles. Clin Gastroenterol Hepatol 7, 966-971.

13. Catamo E, Zupin L, Segat L, et al. (2015) HLA-G and susceptibility to develop celiac disease. Hum Immunol $\mathbf{7 6}$, 36-41.

14. Di Cagno R, De Angelis M, De Pasquale I, et al. (2011) Duodenal and faecal microbiota of celiac children: molecular, phenotype and metabolome characterization. $B M C$ Microbiol 11, 219.

15. Guandalini S (2007) The influence of gluten: weaning recommendations for healthy children and children at risk for celiac disease. Nestle Nutr Workshop Ser Pediatr Program 60, 139-155.

16. Akobeng AK, Ramanan AV, Buchan I, et al. (2006) Effect of breast feeding on risk of coeliac disease: a systematic review and meta-analysis of observational studies. Arch Dis Child 91, 39-43.

17. Lionetti E, Castellaneta S, Francavilla R, et al. (2014) Introduction of gluten, HLA status, and the risk of celiac disease in children. $N$ Engl J Med 371, 1295-1303.

18. Chmielewska A, Pieścik-Lech M, Szajewska H, et al. (2015) Primary prevention of celiac disease: environmental factors with a focus on early nutrition. Ann Nutr Metab 67, 43-50.

19. Harmsen HJ, Wildeboer-Veloo AC, Raangs GC, et al. (2000) Analysis of intestinal flora development in breast-fed and formula-fed infants by using molecular identification and detection methods. J Pediatr Gastroenterol Nutr 30, 61-67.

20. Plot L \& Amital H (2009) Infectious associations of celiac disease. Autoimmun Rev 8, 316-319.

21. Stene LC, Honeyman MC, Hoffenberg EJ, et al. (2006) Rotavirus infection frequency and risk of celiac disease autoimmunity in early childhood: a longitudinal study. Am J Gastroenterol 101, 2333-2340.

22. Pavone $\mathrm{P}$, Nicolini E, Taibi R, et al. (2007) Rotavirus and celiac disease. Am J Gastroenterol 102, 1831-1831.

23. Ivarsson A, Hernell O, Nystrom L, et al. (2003) Children born in the summer have increased risk for coeliac disease. $J$ Epidemiol Community Health 57, 36-39.

24. Lewy H, Meirson H \& Laron Z (2009) Seasonality of birth month of children with celiac disease differs from that in the general population and between sexes and is linked to 
family history and environmental factors. J Pediatr Gastroenterol Nutr 48, 181-185.

25. Camilleri M, Nullens S \& Nelsen T (2012) Enteroendocrine and neuronal mechanisms in pathophysiology of acute infectious diarrhea. Dig Dis Sci 57, 19-27.

26. Monteleone G, Pender SL, Wathen NC, et al. (2001) Interferon- $\alpha$ drives $\mathrm{T}$ cell-mediated immunopathology in the intestine. Eur J Immunol 31, 2247-2255.

27. Cammarota G, Cuoco L, Cianci R, et al. (2000) Onset of coeliac disease during treatment with interferon for chronic hepatitis C. Lancet 356, 1494-1495.

28. Hernandez L, Johnson TC, Naiyer AJ, et al. (2008) Chronic hepatitis $\mathrm{C}$ virus and celiac disease, is there an association? Dig Dis Sci 53, 256-261.

29. Wieser H (2007) Chemistry of gluten proteins. Food Microbiol 24, 115-119.

30. Kontogiorgos V (2011) Microstructure of hydrated gluten network. Food Res Int 44, 2582-2586.

31. Vader LW, Stepniak DT, Bunnik EM, et al. (2003) Characterization of cereal toxicity for celiac disease patients based on protein homology in grains. Gastroenterology 125, 1105-1113.

32. Hausch F, Shan L, Santiago NA, et al. (2002) Intestinal digestive resistance of immunodominant gliadin peptides. Am J Physiol Gastrointest Liver Physiol 283, G996-G1003.

33. De Re V, Caggiari L, Tabuso M, et al. (2013) The versatile role of gliadin peptides in celiac disease. Clin Biochem $\mathbf{4 6}$, 552-560.

34. Fasano A (2012) Intestinal permeability and its regulation by zonulin: diagnostic and therapeutic implications. Clin Gastroenterol Hepatol 10, 1096-1100.

35. Schuppan D, Junker Y \& Barisani D (2009) Celiac disease: from pathogenesis to novel therapies. Gastroenterology 137, 1912-1933.

36. Comerford R, Coates C, Byrne G, et al. (2014) Characterisation of tissue transglutaminase-reactive $\mathrm{T}$ cells from patients with coeliac disease and healthy controls. Clin Immunol 154, 155-163.

37. Hana A, Newell EW, Glanvilled J, et al. (2013) Dietary gluten triggers concomitant activation of $\mathrm{CD}^{4+}$ and $\mathrm{CD}^{8+} \alpha \beta$ T cells and $\gamma \delta$ T cells in celiac disease. Proc Natl Acad Sci US A 32, 13073-13078.

38. Korneychuk N, Ramiro-Puig E, Ettersperger J, et al. (2014) Interleukin 15 and $\mathrm{CD} 4 \mathrm{D} \mathrm{T}$ cells cooperate to promote small intestinal enteropathy in response to dietary antigen. Gastroenterology 146, 1017-1027.

39. Sollid LM, Qiao SW, Anderson RP, et al. (2012) Nomenclature and listing of celiac disease relevant gluten T-cell epitopes restricted by HLA-DQ molecules. Immunogenetics $\mathbf{6 4}$, 455-460.

40. Bodd M, Ráki M, Tollefsen S, et al. (2010) HLA-DQ2restricted gluten-reactive $\mathrm{T}$ cells produce IL-21 but not IL-17 or IL-22. Mucosal Immunol 3, 594-601.

41. Patruno A, Pesce M, Marrone A, et al. (2012) Activity of matrix metallo proteinases (MMPs) and the tissue inhibitor of MMP (TIMP)-1 in electromagnetic field-exposed THP-1 cells. J Cell Physiol 227, 2767-2774.

42. Ciccocioppo R, Di Sabatino A, Bauer M, et al. (2005) Matrix metalloproteinase pattern in celiac duodenal mucosa. Lab Invest 85, 397-407.

43. Bruewer M, Luegering A, Kucharzik T, et al. (2003) Proinflammatory cytokines disrupt epithelial barrier function by apoptosis-independent mechanisms. J Immunol 171, 6164-6172.

44. Lahdenperä A (2011) The effect of gluten-free diet on Th1-Th2-Th3-associated intestinal immune responses in celiac disease. Scand J Gastroenterol 46, 538-549.
45. Tollefsen S, Rentz-Hansen H, Fleckenstein B, et al. (2006) HLADQ2 and -DQ8 signatures of gluten T cell epitopes in celiac disease. J Clin Invest 116, 2226-2236.

46. Muller JR, Waldmann TA, Kruhlak MJ, et al. (2012) Paracrine and transpresentation functions of IL-15 are mediated by diverse splice versions of IL-15R $\alpha$ in human monocytes and dendritic cells. J Biol Chem 287, 40328-40338.

47. Mention JJ, Ben Ahmed M, Bègue B, et al. (2003) Interleukin 15: a key to disrupted intraepithelial lymphocyte homeostasis and lymphomagenesis in celiac disease. Gastroenterology 125, 730-745.

48. Hourigan CS (2006) The molecular basis of coeliac disease. Clin Exp Med 6, 53-59.

49. Meresse B, Chen Z, Ciszewski C, et al. (2004) Coordinated induction by IL15 of a TCR-independent NKG2D signaling pathway converts CTL into lymphokine-activated killer cells in celiac disease. Immunity 21, 357-366.

50. Malamut G, El Machhour R, Montcuquet N, et al. (2010) IL-15 triggers an antiapoptotic pathway in human intraepithelial lymphocytes that is a potential new target in celiac disease-associated inflammation and lymphomagenesis. J Clin Invest 120, 2131-2143.

51. Woodward J (2011) Coeliac disease. Medicine 39, 173-177.

52. Monteleone G, Caruso R, Fina D, et al. (2006) Control of matrix metalloproteinase production in human intestinal fibroblasts by interleukin 21. Gut 55, 1774-1780.

53. Caruso R, Fina D, Peluso I, et al. (2007) A functional role for interleukin-21 in promoting the synthesis of the T-cell chemoattractant, MIP-3 $\alpha$, by gut epithelial cells. Gastroenterology 132, 166-175.

54. De Paolo RW, Abadie V, Tang F, et al. (2011) Co-adjuvant effects of retinoic acid and IL-15 induce inflammatory immunity to dietary antigens. Nature 471, 220-224.

55. Hamer RJ (2005) Coeliac disease: background and biochemical aspects. Biotechnol Adv 23, 401-408.

56. Dewar D, Pereira SP \& Ciclitira PJ (2004) The pathogenesis of coeliac disease. Int J Biochem Cell Biol 36, 17-24.

57. Uygur-Bayramicli O \& Melih Özel A (2011) Celiac disease is associated with neurological syndromes. Dig Dis Sci 56, $1587-1588$

58. Hu WT, Murray JA, Greenaway MC, et al. (2006) Cognitive impairment and celiac disease. Arch Neurol 63, 1440-1446.

59. Arnone JM \& Conti RP (2015) Neuropsychiatric features of celiac disease. Int J Celiac Dis 3, 77-83.

60. Vieira C, Jatobá I, Matos M, et al. (2013) Prevalence of celiac disease in children with epilepsy. Arq Gastroenterol 50, 290-296.

61. Parisi P, Pietropaoli N, Ferretti A, et al. (2015) Role of the gluten-free diet on neurological-EEG findings and sleep disordered breathing in children with celiac disease. Seizure 25, 181-183.

62. Lerner A, Makhoul BF, Eliakim R, et al. (2012) Neurological manifestations of celiac disease in children and adults. Eur Neurol J 4, 15-20.

63. Neto J, Costa A, Magalhaes FG, et al. (2004) Neurological manifestations of celiacs disease. Arq Neuropsiquiatri 62, 969-972.

64. Zelnik N, Pacht A, Obeid R, et al. (2004) Range of neurologic disorders in patients with celiac disease. Pediatrics 113, $1672-1676$.

65. Bushara KO (2005) Neurologic presentation of celiac disease. Gastroenterology 128, S92-S97.

66. Gabrielli M, Cremonini F, Fiore G, et al. (2003) Association between migraine and celiac disease: results from a preliminary case-control and therapeutic study. $A m J$ Gastroenterol 98, 625-629. 
67. Shahriar N (2012) Neurological manifestations, diagnosis, and treatment of celiac disease: a comprehensive review. Iran J Neurol 11, 59-64.

68. Cooke WT \& Smith WT (1966) Neurological disorders associated with adult celiac disease. Brain 89, 6822-6837.

69. Fasano A (2003) Celiac disease: how to handle a clinical chameleon. New Engl J Med 348, 2568-2570.

70. Hadjivassiliou M, Mäki M, Sanders DS, et al. (2006) Autoantibody targeting of brain and intestinal transglutaminase in gluten ataxia. Neurology 66, 373-377.

71. Ludvigsson JF, Zingone F, Tomson T, et al. (2012) Increased risk of epilepsy in biopsy-verified celiac disease: a populationbased cohort study. Neurology 78, 1401-1407.

72. Hadjivassiliou M, Grünewald RA \& Davies-Jones GA (2002) Gluten sensitivity as a neurological illness. J Neurol Neurosurg Psychiatry 72, 560-563.

73. Chin RL, Sander HW, Brannagan TH, et al. (2003) Celiac neuropathy. Neurology 60, 1581-1585.

74. Luostarinen L, Himanen SL, Luostarinen M, et al. (2003) Neuromuscular and sensory disturbances in patients with well treated coeliac disease. J Neurol Neurosurg Psychiatry 74, 490-494.

75. Tursi A, Giorgetti GM, Iani C, et al. (2006) Peripheral neurological disturbances, autonomic dysfunction, and antineuronal antibodies in adult celiac disease before and after a gluten-free diet. Dig Dis Sci 51, 1869-1874.

76. Uzma R, Imdad A \& Beg M (2015) Rare neurological manifestation of celiac disease. Case Rep Gastroenterol 9, 200-205.

77. Chaudhry V \& Ravich WJ (2001) Other neurological disorders associated with gastrointestinal, liver, or pancreatic diseases. Neurol Gen Med 3, 283-284.

78. Issikay S \& Kocamaz H (2015) The neurological face of celiac disease. Arq Gastroenterol 52, 167-170.

79. Ferretti A, Parisi P \& Villa MP (2013) The role of hyperhomocysteinemia in neurological features associated with coeliac disease. Med Hypotheses 81, 524-531.

80. Lichtwark IT, Newnham ED, Robinson SR, et al. (2014) Cognitive impairment in coeliac disease improves on a gluten-free diet and correlates with histological and serological indices of disease severity. Aliment Pharmacol Ther $\mathbf{4 0}$, 160-170.

81. Theoharides C, Stewart JM, Hatziagelaki E, et al. (2015) Brain "fog," inflammation and obesity: key aspects of neuropsychiatric disorders improved by luteolin. Front Neurosci 9, 225.

82. Lurie Y, Landau DA, Pfeffer J, et al. (2008) Celiac disease diagnosed in the elderly. J Clin Gastroenterol 42, 59-61.

83. Casella S, Zanini B, Lanzarotto F, et al. (2012) Cognitive performance is impaired in coeliac patients on gluten free diet: a case-control study in patients older than 65 years of age. Dig Liver Dis 44, 729-735.

84. Collin P, Pirttilä T, Nurmikko T, et al. (1991) Celiac disease, brain atrophy, and dementia. Neurology $\mathbf{4}, 372-375$.

85. Luostarinen L, Pirttilä T \& Collin P (1999) Coeliac disease presenting with neurological disorders. Eur Neurol 42, 132-135.

86. Cicarelli G, Della Rocca G, Amboni M, et al. (2003) Clinical and neurological abnormalities in adult celiac disease. Neurol Sci 24, 311-317.

87. Karwautz A, Wagner G, Berger G, et al. (2008) Eating pathology in adolescents with celiac disease. Psychosomatics 49, 399-406.

88. Niederhofer H \& Pittschieler K (2006) A preliminary investigation of ADHD symptoms in persons with celiac disease. J Atten Disord 10, 200-204.
89. Barcia G, Posar A, Santucci M \& Parmeggiani A (2008) Autism and coeliac disease. J Autism Dev Disord 38, 407-408.

90. Zingone F, Siniscalchi M, Capone P, et al. (2010) The quality of sleep in patients with coeliac disease. Aliment Pharmacol Ther 32, 1031-1036.

91. Carta MG, Hardoy MC, Boi MF, et al. (2002) Association between panic disorder, major depressive disorder and celiac disease: a possible role of thyroid autoimmunity. J Psychosom Res 53, 789-793.

92. Fera T, Cascio B, Angelini G, et al. (2003) Affective disorders and quality of life in adult coeliac disease patients on a gluten-free diet. Eur $J$ Gastroenterol Hepatol 15, $1287-1292$

93. Smith DF \& Gerdes LU (2012) Meta-analysis on anxiety and depression in adult celiac disease. Acta Psychiatr Scand 125, 189-193.

94. Addolorato G, Mirijello A, D'Angelo C, et al. (2008) State and trait anxiety and depression in patients affected by gastrointestinal diseases: psychometric evaluation of 1641 patients referred to an internal medicine outpatient setting. Int J Clin Pract 62, 1063-1069.

95. Addolorato G, Mirijello A, D'Angelo C, et al. (2008) Social phobia in coeliac disease. Scand J Gastroenterol 43, 410-415.

96. Ludvigsson JF, Reutfors J, Osby U, et al. (2007) Coeliac disease and risk of mood disorders - a general populationbased cohort study. I Affect Disord 99, 117-126.

97. Hallert C, Svensson M, Tholstrup J, et al. (2009) Clinical trial: B vitamins improve health in patients with coeliac disease living on a gluten-free diet. Aliment Pharmacol Ther 29, 811-816.

98. Thompson T, Dennis M, Higgins LA, et al. (2005) Gluten-free diet survey: are Americans with coeliac disease consuming recommended amounts of fibre, iron, calcium and grain foods? J Hum Nutr Diet 18, 163-169.

99. Häuser W, Stallmach A, Caspary WF, et al. (2007) Predictors of reduced health-related quality of life in adults with coeliac disease. Aliment Pharmacol Ther 25, 569-578.

100. Delgado P, Price LH, Miller HL, et al. (1994) Serotonin and the neurobiology of depression: effects of tryptophan depletion in drug-free depressed patients. Arch Gen Psychiatry 51, 865-874.

101. Kronfol Z \& Remick DG (2000) Cytokines and the brain: implications for clinical psychiatry. Am J Psychiatry 157, 683-694.

102. Addolorato G, Capristo E, Ghittoni G, et al. (2001) Anxiety but not depression decreases in coeliac patients after one-year gluten-free diet: a longitudinal study. Scand $J$ Gastroenterol 36, 502-506.

103. Garud S, Leffler D, Dennis M, et al. (2009) Interaction between psychiatric and autoimmune disorders in coeliac disease patients in the Northeastern United States. Aliment Pharmacol Ther 29, 898-905.

104. Mazzone L, Reale L, Spina M, et al. (2011) Compliant gluten-free children with celiac disease: an evaluation of psychological distress. BMC Pediatrics 2011, 114-116.

105. Pynnönen PA, Isometsä ET, Aronen ET, et al. (2004) Mental disorders in adolescents with celiac disease. Psychosomatics 45, 325-335.

106. Sharma TR, Kline DB, Shreeve DF, et al. (2011) Psychiatric comorbidities in patients with celiac disease: is there any concrete biological association? Asian J Psychiatr 4, 150-151.

107. van Hees NJM, van der Does W \& Giltay EJ (2013) Coeliac disease, diet adherence and depressive symptoms. J Psychosom Res 74, 155-160. 
108. Urban-Kowalczyk M, Emigielski J, Gmitrowicz A, et al. (2014) Neuropsychiatric symptoms and celiac disease. Neuropsychiatr Dis Treat 10, 1961-1964.

109. Lee A \& Newman JM (2003) Celiac diet: its impact on quality of life. J Am Diet Assoc 103, 1533-1535.

110. Eaton WW, Byrne M, Ewald H, et al. (2006) Association of schizophrenia and autoimmune diseases: linkage of Danish national registers. Am J Psychiatry 163, 521-528.

111. Chen SJ, Chao TL, Chen CY, et al. (2012) Prevalence of autoimmune diseases in in-patients with schizophrenia: nationwide population-based study. Br J Psychiatry 200, 374-380.

112. Singh MM \& Kay S (1976) Wheat gluten as a pathogenic factor in schizophrenia. Science 191, 401-402.

113. Kalaydjian AE, Eaton W, Cascella N, et al. (2006) The gluten connection: the association between schizophrenia and celiac disease. Acta Psychiatr Scand 113, 82-90.

114. Jackson T, Eaton W, Cascella N, et al. (2012) A gluten-free diet in people with schizophrenia and anti-tissue transglutaminase or anti-gliadin antibodies. Schizophr Res $\mathbf{1 4 0}$, 262-263.

115. Zhong F, McCombs CC, Olson JM, et al. (1996) An autosomal screen for genes that predispose to celiac disease in the western counties of Ireland. Nat Genet 14, 329-333.

116. Straub RE, MacLean CJ, O'Neill FA, et al. (1995) A potential vulnerability locus for schizophrenia on chromosome 6p24-22: evidence for genetic heterogeneity. Nat Genet 11, 287-293.

117. Li T, Underhill J, Liu XH, et al. (2001) Transmission disequilibrium analysis of HLA class II DRB1, DQA1, DQB1 and DPB1 polymorphisms in schizophrenia using family trios from a Han Chinese population. Schizophr Res 49, 73-78.

118. Samaroo D, Dickerson F, Kasarda DD, et al. (2010) Novel immune response to gluten in individuals with schizophrenia. Schizophr Res 118, 248-255.

119. Eaton WW, Mortensen PB, Agerbo E, et al. (2004) Coeliac disease and schizophrenia: population based case control study with linkage of Danish national registers. BMJ 328, 438-439.

120. Karlsson H, Blomström A, Wicks S, et al. (2012) Maternal antibodies to dietary antigens and risk for nonaffective psychosis in offspring. Am J Psychiatry 169, 625-632.

121. Mormile R (2016) Schizophrenia in celiac disease: a myth or reality? Int J Colorectal Dis 31, 1085.

122. Severance EG, Yolken RH \& Eaton WW (2016) Autoimmune diseases, gastrointestinal disorders and the microbiome in schizophrenia: more than a gut feeling. Schizophr Res 176, 23-35.

123. Nemani K, Hosseini Ghomi R, McCormick B, et al. (2015) Schizophrenia and the gut-brain axis. Prog Neuropsychopharmacol Biol Psychiatry 56, 155-160.

124. Eaton WW, Chen LY, Dohan FC Jr, et al. (2015) Improvement in psychotic symptoms after a gluten-free diet in a boy with complex autoimmune illness. Am J Psychiatry 172, 219-221.

125. Cakir D, Tosun A, Polat M, et al. (2007) Subclinical neurological abnormalities in children with celiac disease receiving a gluten-free diet. J Pediatr Gastroenterol Nutr $\mathbf{4 5}$, 366-369.

126. De Santis A, Addolorato G, Romito A, et al. (1997) Schizophrenic symptoms and SPECT abnormalities in a coeliac patient: regression after a gluten-free diet. J Intern Med 242, 421-423.

127. Casellas F, Rodrigo L, López Vivancos J, et al. (2008) Factors that impact health-related quality of life in adults with celiac disease: a multicenter study. World J Gastroenterol 14 $46-52$.

128. Khoshbaten M, Rostami Nejad M, Sharifi N, et al. (2012) Celiac disease in patients with chronic psychiatric disorders. Gastroenterol Hepatol Bed Bench 5, 90-93.

129. Casellas F, Rodrigo L, Lucendo AJ, et al. (2015) Benefit on health-related quality of life of adherence to gluten-free diet in adult patients with celiac disease. Rev Esp Enferm Dig 107, 196-201.

130. Sainsbury K, Mullan B \& Sharpe L (2013) Reduced quality of life in coeliac disease is more strongly associated with depression than gastrointestinal symptoms. J Psychosom Res 75, 135-141.

131. Kukla U (2015) Mental disorders in digestive system diseases - internist's and psychiatrist's insight. Pol Merkur Lekarski 38, 245-249.

132. González-Arancibia C, Escobar-Luna J \& Barrera-Bugueño C (2016) What goes around comes around: novel pharmacological targets in the gut-brain axis. Ther Adv Gastroenterol 9, 339-353.

133. Julio-Pieper M, Bravo JA, Aliaga E, et al. (2014) Intestinal barrier dysfunction and central nervous system disorders a controversial association. Aliment Pharmacol Ther 40, 1187-1201.

134. Petra A, Panagiotidou S, Hatziagelaki E, et al. (2015) Gut-microbiota-brain axis and its effect on neuropsychiatric disorders with suspected immune dysregulation. Clin Ther 37, 984-995.

135. Liu X, Cao S \& Zhang X (2015) Modulation of gut microbiotabrain axis by probiotics, prebiotics, and diet. J Agric Food Chem 63, 7885-7895.

136. Slyepchenko A, Carvalho AF, Cha DS, et al. (2014) Gut emotions - mechanisms of action of probiotics as novel therapeutic targets for depression and anxiety disorders. CNS Neurol Disord Drug Targets 13, 1770-1786.

137. Sherwin E, Sandhu KV, Dinan TG, et al. (2016) May the force be with you: the light and dark sides of the microbiota-gutbrain axis in neuropsychiatry. CNS Drugs 30, 1019-1041.

138. Moos WH, Faller DV, Harpp DN, et al. (2016) Microbiota and neurological disorders: a gut feeling. Biores Open Access 5, 137-145.

139. Collado MC, Donat E, Ribes-Koninckx C, et al. (2009) Specific duodenal and faecal bacterial groups associated with paediatric coeliac disease. J Clin Pathol 62, 264-269.

140. Nistal E, Caminero A, Vivas S, et al. (2012) Differences in faecal bacteria populations and faecal bacteria metabolism in healthy adults and celiac disease patients. Biochimie $\mathbf{9 4}$, $1724-1729$.

141. Marasco G, Di Biase AR, Schiumerini R, et al. (2016) Gut microbiota and celiac disease. Dig Dis Sci 61, 1461-1472.

142. Carabotti C, Scirocco A, Maselli MA, et al. (2015) The gut-brain axis: interactions between enteric microbiota, central and enteric nervous systems. Ann Gastroenterol 28, 203-209.

143. Galland L (2014) The gut microbiome and the brain. J Med Food 17, 1261-1272.

144. Bella R, Lanza G, Cantone M, et al. (2015) Effect of a glutenfree diet on cortical excitability in adults with celiac disease. PLOS ONE 10, e0129218.

145. Pennisi G, Lanza G, Giuffrida S, et al. (2014) Excitability of the motor cortex in de novo patients with celiac disease. PLOS ONE 9, e102790. 